\title{
Dual vs. single encoding in recognition memory as a function of lag, delay, and availability of semantic information
}

\author{
BRUCE EARHARD and DENYSE LANDRY \\ Dalhousie University, Halifax, Nova Scotia, Canada BsH $4 \mathrm{~J} 1$
}

\begin{abstract}
One objective of the study was to assess the view of Winograd and Raines (1972) that the absence of a difference between dual as opposed to single encodings of a word at long lags may be due to the fact that subjects can establish two independent temporal tags when a to-be-remembered (TBR) word is presented at long lags. Winograd and Raines' argument that the effectiveness of any such temporal tags should diminish over time was examined. A second objective was to determine whether providing information about the semantic senses used during encoding operations would facilitate recognition, as "sense tagging" theories require. Words were presented twice in either the same low-frequency context or the same high-frequency context, or once in both a high- and low-frequency context at Lags 0 and 15 . A recognition test was given immediately, or after a 1-week delay. The test was either context free or each word was accompanied by phrases which, in the case of TBR words, cued subjects with respect to the semantic senses of the word used in initial encoding. All manipulations influenced performance, but not-in accordance with theoretical expectations.
\end{abstract}

It is frequently assumed that the establishment of more than one memory trace of an event can facilitate retention. The argument normally advanced is that if access to one trace is blocked or closed at time of retention, other avenues of access exist (e.g., Melton, $1967,1970)$. While such arguments have substantial intuitive appeal, a variety of experimental efforts to determine whether the establishment of two semantic traces offers any advantage over the establishment of a single semantic trace have had a a very limited success. Dual encoding has been shown generally to be superior only under conditions where the to-be-rememberd (TBR) events follow each other in close temporal sequence. If a delay or lag is introduced between successive presentations, dual semantic encoding offers little advantage over two single presentations which maintain the same semantic sense in studies using either free recall (e.g., Madigan, 1969) or recognition procedures (Winograd \& Raines, 1972). The present study is concerned with determining whether any variation in this generally observed pattern can be produced by manipulating semantic and temporal variables.

One manipulation undertaken stems from a proposal made by Winograd and Raines (1972). Heavily influenced by the work of Hintzman and Block (1971) which indicates that subjects can maintain a very accurate temporal record of the sequence of verbal material presented for study, Winograd and Raines proposed that, when presentations of TBR items are separated by a long lag, subjects encode the trace at two separate

This research was supported by Grant A 0142 from the National Research Council of Canada to B. Earhard. We are grateful to M. F. Geis and E. Winograd for providing a list of homographs. temporal locations, $t_{1}$ and $t_{2}$, and they can use information stored at either temporal location to determine whether a word has been previously presented. At short intervals, where items are presented in close temporal proximity, it is assumed that the temporal grain is not sufficiently fine to permit the establishment of two separate and discriminable traces without a great deal of difficulty. The main point is that at long lags differential or dual encoding along the temporal dimension occurs, and the advantage gained by the existence of two different temporal encodings is assumed to be comparable to that gained by differential semantic encoding.

One way to determine whether the high level of performance observed when two representations of a TBR word are separated by a long lag reflects a form of dual temporal encoding is to introduce a long delay between presentation of the TBR words and the retention test. As Winograd and Raines (1972) note, a very long delay between presentation and test should blur the temporal grain and make any separate temporal traces established at long lags within a relatively short 10 -min presentation sequence hard to discriminate, thus eliminating, or at least reducing, the lag effect. It would be expected, therefore, that, with the introduction of a long delay between presentation and test, encoding TBR words in two different semantic senses would result in better performance than encoding TBR words in the same semantic sense at both long and short lags. Indeed, in the only recall study which has investigated the use of multiple encoding with long time intervals separating presentation and test, it was found that the effect of utilizing more than one code was most pronounced at long time intervals (Bevan, Dukes, \& Avant, 
1966). No such experiments have been carried out using recognition procedures.

A second objective was to determine whether the limited advantage associated with the establishment of more than one memory trace could be due to the possibility that subjects do not effectively examine all the various semantic senses of a word at the time retention is tested. There is ample justification for such concern. Until recently, recognition was viewed as a rather straightforward mechanical process. If a TBR word was presented on a recognition test, it was assumed that a subject had immediate access to the stored memory trace of that word and could judge on the basis of available contextual or temporal markers (Anderson \& Bower, 1972) whether or not the word had been previously presented. Research carried out by Light and Carter-Sobell (1970) and Tulving and his associates (e.g., Tulving \& Thomson, 1973) indicate the situation is not so simple. Their data make it extremely difficult to assume that a central generic memory trace is activated and immediately accessed. To the contrary, the data now available suggest that a word has many senses or meanings (Martin, 1975) and that all these senses are not mechanically accessed at recall. If, for example, a subject encodes the word "bank" in the sense of a place where money is stored, he will very likely not recognize the word as having been previously encountered if he, for some reason, thinks of the word "bank" in the sense of the side of a river at the time of the retention test. Contemporary formulations of the recognition process have been modified to take into consideration the problem of multiple senses or meanings; a number of theoretical accounts now assume that the primary problem in recognition is gaining access to a particular semantic sense on presentation of a word and then proceeding from that semantic sense to associated list markers or contextual propositions to determine if that particular sense of the word occurred in a presentation sequence (Anderson \& Bower, 1974; Reder, Anderson, \& Bjork, 1974). What is important to note is that it is assumed that one proceeds from a semantic meaning or sense to occurrence or contextual information which is used to judge whether or not a word has been presented. If this is the case, then acquainting subjects at the time of retention with the different senses used in encoding should markedly facilitate performance. Further, if the TBR words are encoded in both a relatively high- and a relatively lowfrequency context, it should be possible to clarify some subordinate issues of interest.

One issue of interest is whether information about relevant semantic senses on the recognition test will affect words encoded in high- and low-frequency context differently. Winograd and Raines (1972) report that TBR words presented in a high-frequency context are recognized with greater accuracy than words presented at low-frequency contexts. They attribute this difference to subjects' disposition to consult the culturally more dominant semantic senses at time of re- tention. It follows, therefore, that subjects presented TBR words in low-frequency contexts, and then provided with information about semantic senses used during encoding, should profit more from this information than subjects presented TBR words in highfrequency culturally dominant senses. A second issue of interest is whether anticipated differences resulting from initially encoding TBR words in low- and highfrequency contexts will be magnified by the introduction of a substantial delay between presentation and test. It would seem reasonable to expect that subjects would be even more likely to revert to culturally dominant (and inappropriate) semantic senses in undertaking an uncued recognition test after a long delay than if the test was undertaken immediately.

\section{METHOD}

\section{Design}

Four main variables were manipulated: context, lag, time of test, and type of test. Each TBR word appeared twice in the presentation sequence. The second presentation occurred at Lag 0 for half of the words and at Lag 15 for the other half of the words. The assignment of words to the two lag conditions was counterbalanced in each of three distinctive context conditions. In the "different" (D) context condition, each TBR word appeared once in a high-frequency semantic context and once in a low-frequency semantic context, with the order of presentation of the two different semantic contexts counterbalanced at both lag intervals. In the "high" $(H)$ context condition, the TBR word appeared twice in the same high-frequency context; in the "low" (L) condition, the TBR word appeared twice in the same low-frequency sense. Subjects were given a recognition test either immediately following the presentation sequence or 1 week later. The recognition test was either cued or noncued. In the noncued test both TBR words and distractors appeared without context. In the cued test each TBR word and each distractor was accompanied by two brief phrases, one providing a high-frequency and the other a low-frequency semantic sense of the word. In the case of the TBR words, the semantic sense described corresponded to that conveyed by the sentences used as context during the initial presentation.

\section{Materials}

One hundred and sixty homographs, all having an $\mathrm{A}$ or $\mathrm{AA}$ frequency rating according to the Thorndike-Lorge word count, were used as TBR words, filler words, and distractor words. An additional seven words of mixed frequency were provided for use as primacy and recency buffer items. The words used were drawn from the list of homographs assembled by Winograd and his associates (Winograd \& Geis, 1974; Winograd \& Raines, 1972). In the Winograd and Raines study, three familiar but different semantic senses were selected and ranked for frequency of usage. In the present study, the highest and lowest ranked semantic senses were chosen for use in establishing highand low-frequency contexts. Homographs were incorporated into sentences to convey the required high- or low-frequency sense. The sentence frames used by Winograd and Raines (1972) were retained for this purpose, but in six instances it was necessary to replace an American referent with a local Canadian referent in order to make a particular word sense clearer to a Canadian subject population. As will be seen, the modifications introduced had no apparent influence on frequency designations. The large difference anticipated when TBR words were presented in high- as opposed to low-frequency contexts was very evident.

The materials were assembled into booklets for presentation to subjects. A single sentence was typed on each $81 / 2 \times 11$ in. 
page of the booklet. Within each sentence, the TBR word or the filler word was underlined and printed in capital letters. In addition to the $48 \mathrm{TBR}$ items and 16 filler words used to maintain the required spacings within the presentation sequence for words presented at different lags, four homographs in sentence context served as primacy buffer items and three served as recency buffers. Thus, a completed booklet contained 119 pages plus a cover page with instructions. The 119 pages of context represent a presentation sequence composed of 48 TBR items presented twice in either the same or different contexts (24 at Lag 0 and 24 at Lag 15) and 23 filler and buffer items. There were three different types of booklets: one for the $D$ condition, one for the $H$ condition, and one for the $L$ condition; each type of booklet had four different forms in which TBR words were presented in different orders.

The recognition test was a three-alternative forced choice type. In the noncued test each of the TBR words appeared on a separate line with two distractor items. Distractor words were assigned to TBR words at random, with the restriction that no three alternatives should appear together in more than one test form. The relative position of the TBR word and distractor words varied randomly from line to line, and subjects were required to designate one word in each line as the TBR word. A total of six different test forms were constructed.

On the cued recognition test, immediately to the left of each of the 48 TBR homographs, were two short phrases which reflected the high and low-frequency semantic contexts used during input, e.g.:

\section{Master possessing expert skills \\ form of address to a child}

Homographs used as distractors were also accompanied by two short phrases reflecting high-and low-frequency semantic senses. High- and low-frequency senses used to accompany distractor words were obtained by consulting the Barnhart's (1959) American College Dictionary, which lists semantic senses of a word in order of usage according to the Lorge-Thorndike semantic count. The first listed semantic sense was used as a highfrequency sense was selected by drawing at random one of the remaining word senses. Phrases conveying the required word senses were constructed, and altered if necessary, until three independent judges were in accord about the clarity and precision of the semantic sense conveyed by the phrases. Distractor words were assigned to TBR words at random, with the restriction that no combination of a TBR word and two distractors could appear together in more than one test form. There were six different test forms. In the test booklet, each test set (composed of a TBR word, two distractors, and accompanying phrases) was separated by dotted lines, and subjects were required to designate one of the three words (i.e., either a TBR word or a distractor) as being correct. An equal number of words presented at $\operatorname{Lag} 0$ and $\operatorname{Lag} 15$ were contained in each half of both cued and noncued test forms.

\section{Procedure and Subjects}

The experiment was carried out during part of the normal lecture period of four very large undergraduate classes in psychology. Two classes were randomly selected to be given a delayed recall test and the other two classes were given an immediate recall test after presentation of the TBR materials. Prior to the arrival of students, booklets containing presentation and test materials were arranged on desks in each row in random order, with two restricitons: each of the different forms of various conditions had to be represented in a row before that form could be repeated and the same form could not be assigned to adjacent seats. Subjects were required to fill the room in order of arrival row by row, thus assuring that approximately equal numbers of subjects were assigned to all conditions. Subjects in all conditions were informed that we were undertaking a test to determine how well they could remember words. They were told that a sentence would appear on each page of the booklet in front of them and that some sentences might appear more than once. They were instructed that the retention test would involve the underlined and capitalized word in each sentence, and that they were to read each sentence carefully, and then reread the word in the time provided. They were allowed $5 \mathrm{sec}$ to read each page before being required to turn to the next page. A Hunter Klockounter was used to time the 5 - sec interval, and the instruction to turn the page was delivered over a loudspeaker system. Two assistants moved about the class to insure pageturning instructions were followed.

After the entire set of sentences had been presented, subjects were asked to write on a condition-coded sheet of paper an estimate of the proportion of the words presented they thought they would be able to remember. Subjects in the two classes to be given delayed recognition tests were informed that the purpose of the study was to see how closely subjects were able to estimate their recall capability and that their estimates would be compared with the actual recall capabilities of two other classes which would be given an immediate memory test. The sheets upon which the estimates were written were then collected and the lecture commenced. Subjects given an immediate retention test were asked, after making their recall estimate, to pick up the test booklets beside their seat and to read the instructions and commence the test. In the case of the uncued retention test, there were 48 lines, each containing three words; subjects were required to circle the one word on each line they thought had been previously presented. In the case of the cued retention test, instructions on the first page informed subjects that there were two meanings contained in phrases beside each word, and an illustrative example was provided to show how each set of three words (i.e., a TBR word and two distractors) would be arranged on a page. They were informed that they were to read carefully the phrases beside each word because the meanings conveyed by these phrases would help them to decide which of the three words in a given set had been previously encountered. They were required to circle one, and one word only, from each set of three words. Neither retention test was paced.

Subjects in the two classes to be given a delayed retention test were asked 1 week after being given the initial test to obtain from the experimenter and assistants a recall booklet with their name on it, and told to proceed in the same manner as subjects given the immediate retention test to complete the test after reading the instructions. The assignment of appropriate recognition test booklets to subjects was achieved by using the code on the sheets they had signed the week before containing an estimate of the proportion of words they thought they could recall. After completion of the retention test, the purpose of the experiment was briefly described and the lecture began.

As would be expected with such an arrangement, variations in class size and absenteeism combined to leave more subjects than required in some conditions and fewer than required in others. In order to equalize the number of subjects in all conditions, an additional 19 naive subjects were drawn from the same population and randomly assigned to deficient conditions. These subjects were tested in small groups. No more than three subjects were added to any one of the 12 experimental groups by this process. Twenty-four surplus subjects were deleted by means of a table of random numbers prior to any data analysis. By these procedures we obtained a total of 336 subjects, with an equal number of subjects in each of the three different context input conditions, $\mathrm{D}, \mathrm{H}$, and $\mathrm{L}$ : Within each of these conditions an equal number of subjects received either a cued or noncued retention test, and an equal number either an immediatc or a delayed recognition test. 
Table 1A

Mean Recognition Scores Corrected for Guessing are Shown Separately for Subjects in D, H, and L Conditions at Lags 0 and 15 Having Cued vs. Noncued Recognition Tests and Immediate vs. Delayed Recognition Tests

\begin{tabular}{|c|c|c|c|c|c|c|c|}
\hline \multicolumn{4}{|c|}{ Cued Recognition } & \multicolumn{4}{|c|}{ Noncued Recognition } \\
\hline Time of Test & Condition & Lag 0 & Lag 15 & Time of Test & Condition & Lag 0 & Lag 15 \\
\hline Immediate & D & 20.43 & 20.64 & Immediate & $\mathrm{D}$ & 19.13 & 18.96 \\
\hline Delayed & D & 9.59 & 10.02 & Delayed & $\mathrm{D}$ & 8.41 & 8.09 \\
\hline Immediate & $\mathrm{H}$ & 17.66 & 18.59 & Immediate & $\mathbf{H}$ & 16.93 & 18.07 \\
\hline Delayed & $\mathrm{H}$ & 9.00 & 9.54 & Delayed & $\mathrm{H}$ & 7.23 & 10.77 \\
\hline Immediate & L & 17.04 & 18.54 & Immediate & $\mathrm{L}$ & 15.91 & 16.82 \\
\hline Delayed & $\bar{L}$ & 6.91 & 6.70 & Delayed & $\mathrm{L}$ & 6.75 & 6.21 \\
\hline
\end{tabular}

\section{RESULTS}

Individual data sheets were scored and the results tabulated with and without a correction for guessing $[R-W /(n-1)]$ applied to the data of individual subjects (see Murdock, 1963). Table 1 provides an overview of the performance level of subjects at Lag 0 and Lag 15 in each of the 12 different groups used in the experiment. Table $1 \mathrm{~A}$ shows the data corrected for guessing, and Table $1 \mathrm{~B}$ shows the noncorrected data. An analysis of variance was applied to both corrected and noncorrected data. In these analyses Lag was treated as a within factor, and Encoding Context, Semantic Cueing vs. No Cueing at test, and Immediate vs. Delayed test were treated as between factors. As would be expected from an examination of the corrected and noncorrected data provided in Table 1 , the results of the two analyses were very similar, in our consideration of the data and analyses to follow, we will restrict discussion to corrected measures, making note at the appropriate point of the only instance where there was any evidence of a difference in the results of the two analyses.

The first observation that can be made is that varying the character of sentences used as contexts for TBR words has a pronounced effect on recognition memory. Subjects in the D condition had a higher mean level of performance than subjects in the $\mathrm{H}$ and $\mathrm{L}$ conditions at Lag 0 (14.39 vs. 12.70 and 11.65 words, respectively) and at Lag 15 (14.43 vs. 14.24 and 12.07 words, respectively). The overall analysis of variance confirmed that Input Context reliably affected recognition memory $\left[F(2,324)=10.89, \mathrm{p}<.01, \mathrm{MS}_{\mathrm{e}}=34.20\right]$. Preplanned orthogonal comparisons between the $\mathrm{D}$ condition, in which subjects received a TBR word in both a highand a low-frequency context, and the combined means of the $\mathrm{H}$ and $\mathrm{L}$ conditions, in which subjects received either two identical high-frequency or two identical low-frequency context presentations of a TBR word, shows the $\mathrm{D}$ condition to be superior to both Lag 0 and $\operatorname{Lag} 15[\mathrm{t}(324)=6.16, \mathrm{p}<.01$, and $\mathrm{t}(324)=3.55$, $\mathrm{p}<.01$, respectively, $\mathrm{SE}=.359$ ]. These results are relatively unique in indicating an overall advantage for the $D$ condition. Previous research has not shown an overall superiority for dual encoding except at short lags [see Winograd \& Raines (1972) for a consideration of this issue]. The existence of the effect in the present experiment likely reflects design sensitivity which results from the extremely large number of subjects (112) in the three major conditions.

Although the results of the above analyses show the mean performance of subjects in the $D$ condition to be superior to the combined mean performance of subjects in the $\mathrm{H}$ and $\mathrm{L}$ conditions, a more detailed inspection of the data shows that the differences among the $\mathrm{D}, \mathrm{H}$, and $L$ conditions are not uniform at $\operatorname{Lag} 0$ and $\operatorname{Lag} 15$. Only in the case of the $D$ and $L$ conditions is the difference approximately equal at Lag 0 and Lag 15 (14.39 vs. 11.65 and 14.43 vs. 12.07 words, respectively). In the case of the $\mathrm{D}$ and $\mathrm{H}$ conditions, the difference in favor of the $D$ condition is substantial at Lag 0 , but negligible at Lag 15 (14.39 vs. 12.70 and 14.43 vs. 14.24 words, respectively). As far as the $\mathrm{H}$ and $\mathrm{L}$ conditions are concerned, the main difference is at Lag 15 as opposed to Lag 0 (14.24 vs. 12.07 and 12.70 vs. 11.46 words, respectively). The overall analysis of

Table 1B

Mean Recognition Scores Not Corrected for Guessing are Shown Separately for Subjects in D, H, and L Conditions at Lags 0 and 15 Having Cued vs. Noncued Recognition Tests and Immediate vs. Delayed Recognition Tests

\begin{tabular}{|c|c|c|c|c|c|c|c|}
\hline \multicolumn{4}{|c|}{ Cued Recognition } & \multicolumn{4}{|c|}{ Noncued Recognition } \\
\hline Time of Test & Condition & Lag 0 & Lag 15 & Time of Test & Condition & Lag 0 & Lag 15 \\
\hline Immediate & D & 21.57 & 21.68 & Immediate & D & 20.82 & 20.64 \\
\hline Delayed & D & 14.32 & 14.64 & Delayed & D & 13.39 & 13.39 \\
\hline Immediate & $\mathbf{H}$ & 19.75 & 20.39 & Immediate & $\mathbf{H}$ & 19.29 & 20.04 \\
\hline Delayed & $\mathbf{H}$ & 14.00 & 14.21 & Delayed & $\mathbf{H}$ & 12.75 & 15.18 \\
\hline Immediate & $\mathbf{L}$ & 19.36 & 19.96 & Immediate & L & 18.61 & 19.21 \\
\hline Delayed & $\mathbf{L}$ & 12.54 & 12.32 & Delayed & $\mathbf{L}$ & 12.43 & 12.11 \\
\hline
\end{tabular}


variance confirmed the presence of a Lag by Condition interaction $\left[F(2,324)=3.52, \mathrm{p}<.05, \mathrm{MS}_{\mathrm{e}}=9.64\right]$. Application of the Duncan multiple range test (DMRT) showed that the $\mathrm{D}$ and $\mathrm{H}$ conditions were superior to the $\mathrm{L}$ condition at both $\mathrm{Lag} 0$ and $\operatorname{Lag} 15$ at the 5\% level of significance, but that the $\mathrm{H}$ condition differed from the $\mathrm{D}$ condition only at $\mathrm{Lag} 0$. Thus, as has been observed previously in recall (Madigan, 1969) and in recognition studies (Winograd \& Raines, 1972), the main advantage of dual semantic context encoding over single semantic context encoding occurs when the TBR words are presented in close temporal sequence, i.e., at Lag 0 . At longer lags, the advantage of dual encoding is much diminished, and two identical high-frequency encodings do not differ from that observed in the $\mathrm{D}$ condition, where separate high- and low-frequency contexts are used.

If attention is now shifted to the lag effect per se, it is apparent from the data pattern in Table 1 that there is very little evidence of a lag effect in either the $D$ or the $\mathrm{L}$ condition. Application of the DMRT showed that the difference in performance at Lag 0 and $\operatorname{Lag} 15$ was not reliable in the $\mathrm{D}$ or $\mathrm{L}$ condition, whereas in the $\mathrm{H}$ condition there was a large difference in performance which was reliable at the $5 \%$ level of significance. The absence of a lag effect in the D condition was expected in that it is generally concluded that such differential encoding should eliminate the lag effect (Hintzman, 1974; Madigan, 1969). The absence of a lag effect in the $\mathrm{L}$ condition was unexpected, however. Hintzman (1974) has emphasized the ubiquity of the lag effect, and it is surprising that it is not evident with the low-frequency contexts used in the present study especially in view of the large number of subjects employed. Reference to Figure 1 in the Winograd and Raines (1972) study, which used very similar procedures to those employed in the present study, show the effect of lag to be somewhat less extreme in low-frequency contexts, but there was no evidence of an interaction between the $\mathrm{H}$ and $\mathrm{L}$ conditions in that study. All that can be said at this point is that the relationship between the lag effect and low-frequency encoding contexts merits further examination.

While the lag effect was not evident in the $\mathrm{L}$ condition, a pronounced effect was evident in the $\mathrm{H}$ condition, and this allows us to consider one of the two major questions the experiment sought to answer. The question is whether the very substantial improvement at Lag 15 could be attributable to the possibility that subjects can establish two different temporal traces in memory if identical presentations of a TBR word are sufficiently separated during the presentation sequence. Under such circumstances, subjects would have one of the advantages of subjects in the $D$ condition, i.e., the presence of two independently accessible memory traces for use in determining whether a word on the recognition test had been previously presented. It follows, as Winograd and Raines (1972) argue, that if a substantial delay is introduced between presentation and test, fine temporal distinctions should blur and the subject's capacity to utilize this information should diminish. In short, the lag effect should become less pronounced and the advantage of dual semantic encoding should become evident at both Lag 0 and Lag 15 .

The introduction of a 1-week delay between presentation of the TBR words and the recognition test did have a dramatic effect on recognition memory. Mean performance of subjects in the immediate recognition test (averaged over different input contexts, lag, and cued vs. noncued test conditions) was 18.23 words, but after a delay of 1 week, performance dropped to 8.27 words. This difference is highly reliable $[F(1,324)=$ $487.14, \mathrm{p}<.001, \mathrm{MS}_{\mathrm{e}}=34.20$ ], but what is important is that, contrary to expectations, there was no interaction between the length of Time Interval separating presentations of TBR words and $\mathrm{Lag}\left(\mathrm{F}<1, \mathrm{M} \mathrm{SS}_{\mathrm{e}}=\right.$ 9.64), nor was the Lag by Input Context by Immediate vs. Delayed Recognition test interaction reliable $\left[F(2,324)=2.47, p>.05, M S_{e}=9.64\right]$. In fact, the effects of Immediate vs. Delayed Recognition test did not interact reliably with any other variable. It is quite evident that the Winograd and Raines (1972) hypothesis is not supported. The lag effect is not altered even if a very substantial delay is introduced between presentation and test.

A second major objective of the paper was to determine whether informing subjects about the relevant semantic senses used during encoding influenced recognition memory performance. As mentioned earlier, there is ample justification for assuming that subjects neglect to scan all possible semantic meanings when a word is presented for recognition and, rather, seize upon the culturally dominant meaning (Winograd \& Raines, 1972). It would seem reasonable, therefore, to expect that forcing subjects to consider all possible relevant semantic senses should facilitate performance, especially in the $D$ and $L$ conditions. Reference to Table 1 shows that overall performance of subjects informed about the semantic senses used in encoding was higher than that of subjects not informed, but the magnitude of the effect was small. The mean level of performance of subjects not given semantic information on recognition and test trials (averaged over different context, lags, and delay conditions) was 12.77 words, and that of subjects given semantic information was only 13.72 words. This effect just achieved significance in the analysis of corrected data $\left[F(1,324)=4.40, p<.05, \mathrm{MS}_{\mathrm{e}}=34.20\right]$, but fell short of significance in the analysis of noncorrected data $\left[\mathrm{F}(1,324)=3.35, \mathrm{p}<.10, \mathrm{MS}_{\mathrm{e}}=16.55\right]$. This is the only instance where there was any indication of a difference in the analysis of corrected and noncorrected data. Given the marginal significance of the effect of semantic cueing in the case of corrected data, the absence of a difference in the case of noncorrected data, and the extremely small magnitude of observed difference, it seems clear that providing subjects with semantic information on the recognition test had much less effect on performance than would be expected on 
the basis of theoretical accounts which assume that the critical first step in a recognition process is accessing the appropriate word sense. Further, none of the interactions which were expected to result from providing vs. not providing semantic information on the recognition test were evident in any analysis. Contrary to what would be expected if, as has been assumed, subjects revert to more common semantic senses at time of test, the subjects in the $\mathrm{D}$ and $\mathrm{L}$ conditions, who were presented TBR words in less frequent senses, did not profit more from the provision of semantic information than subjects presented TBR words in a high-frequency context. The interaction between input context and providing or not providing semantic information on the recognition test did not even approach significance $\left(\mathrm{F}<1, \mathrm{MS}_{\mathrm{e}}=34.20\right)$. There was also no evidence that at longer delays subjects presented TBR words in low-frequency contexts gained from the provision of semantic information when a long interval separated the presentation sequence and the recognition test. Again the relevant interaction was less than one $\left(\mathrm{MS}_{\mathrm{e}}=34.20\right)$. The overall pattern of the data may be summarized by observing that no interaction involving provision or lack of provision of semantic information on the receognition test approached significance.

\section{DISCUSSION}

Our introduction of a time interval between presentation of the TBR material and the retention test, and the provision of information about relevant semantic senses used in encoding at the time of the recognition test, reliably influenced recognition memory. The way in which recognition memory was influenced, however, did not conform with the theoretical formulations which prompted the research.

Winograd and Raines (1972) argue that better performance observed at long lags when TBR words are presented in the same context reflects the fact that at long lags subjects can establish two traces at separate temporal locations for a TBR word; thus, when retention is tested, if access to one trace is blocked, a second trace is available, just as would be the case in the situation where two different semantic traces of a TBR word are established at input.

The idea itself is a reasonable one. In a review of various explanations of the lag effect, Hintzman (1974) argues cogently that dual encoding interpretations of the lag effect are likely to prove viable only if it can be demonstrated that nonsemantic components of experience are employed in the encoding process. The possibility that the temporal tags could be used is quite real. It is well known that subjects can make effective use of time tags in making frequency judgments (Hintzman \& Block, 1971), and that the availability of frequency information is highly correlated with recognition accuracy (e.g., Underwood, Zimmerman, \& Freund, 1971). Moreover, the accuracy of frequency judyments, and the time tags underlying such judgments, do diminish over long delays, as Winograd and Raines (1972) note. Underwood, Zimmerman, and Freund (1971) present evidence to indicate that, after a delay of a week, subjects are no more accurate in discriminating an item presented twice during input from a new item than are subjects presented an item only once. Thus, in the case of the experiment reported, a l-week delay should have eliminated, or at least significantly reduced, the lag effect-reflecting the fact that two separate time tags were established and available for reference at Lag 15 , but only one at Lag 0 . The data do not support the time tag hypothesis. Nevertheless, in examining the hypothesis, something of interest has been learned about the lag effect. The fact that the lag effect was not altered by a long delay separating presentation and test indicates that the lag effect involves a permanent and enduring change in the memory trace, and that it is unlikely that differential temporal tagging plays an important part in its production.

Further, although we would not like to press the point too strongly, in view of the fact that the experiment was not designed to assess the possibility, it would seem that these results render less tenable any more general encoding variability hypothesis based on nonsemantic factors. For example, Hintzman (1974) notes a possible general interpretation of the lag effect: Elements from the stream of consciousness (which would include time tags) are associated with the internal trace of a TBR word, and at long lags it is more likely that different elements from the stream of consciousness are sampled than at short lags. It follows that this additional tagged information should offer subjects an advantage in making recognition judgments on an immediate recognition test. We would argue, however, that just as time tags become less distinctive as the interval between presentation and test increases, any fine variations in the elements of the stream of consiousness it may be possible to tag at long, as opposed to short, lags within a paced 10-min presentation sequence should become harder to discriminate and utilize as the delay between presentation and test is increased. The end result should be a reduction in the lag effect which is simply not evident in the data.

Shifting attention to the effect of providing information about the semantic senses at time of the recognition test, it seems that what Watkins and Tulving $(1975$, p. 28) term "sense tagging" models of the recognition process receive little support from the data. The essence of the Watkins and Tulving (1975) and Light, Kimble, and Pellegrino (1975) approach points out that occurrence or context information is stored in semantic memory. To get access to occurrence information that permits determination of whether a word has been previously encountered, it is necessary to first reach the appropriate word sense. Reder, Anderson, and Bjork (1974), who are representative of this approach, are quite specific on this point. They argue that "word senses are the basis of recognition," and point out that the word sense generated at the time of the retention 
test must be utilized in encoding if a correct recognition judgment is to be rendered (Reder et al., 1974, p. 55). If accessing the appropriate word sense is a necessary prerequisite for correct recognition, providing subjects with the appropriate semantic sense during the course of the recognition test should result in a profound improvement in performance. The overall improvement observed in the experiment was, on the average, less than a word, and just attained statistical reliability when data were corrected for guessing. Further, a "sense tagging" account requires that the effect of providing semantic information be much greater in the case of words presented in low-frequency contexts than in highfrequency contexts, because subjects are more likely to access the more dominant high-frequency context on a recognition test (Perfetti \& Lindsey, 1974; Winograd \& Raines, 1972). This pattern was also not evident in the data. In passing, it is worth noting that performance observed when TBR words were presented in a lowfrequency context in the present experiment and in the Winograd and Raines study presented an interesting contrast to the typically observed superiority of lowfrequency words in recognition studies (e.g., McCormack \& Swenson, 1972). The finding that it remains evident even when subjects are provided with information about relevant semantic senses on the recognition test raises the possibility that it is not merely failure to reach an appropriate word sense that constitutes the major problem, as generally assumed. Rather, it may be the case that subjects have more difficulty, or perhaps are less efficient, in encoding and retrieving words presented in a low-frequency context.

On the whole, the data obtained appear to be much more compatible with the Tulving and Thomson (1973) encoding specificity hypothesis which assumes that contextual or occurrence information is stored in an episodic memory system that is quite independent of the permanent semantic information store. Semantic information is involved only to the degree that semantic aspects are encoded "as part of the trace of the memory event" (Tulving \& Thomson, 1973, p. 359). That is, recognition memory is assumed to be governed by the availability of the contextual or occurrence information stored quite independently of semantic memory, and activation of a semantic sense does not guarantee access to occurrence or contextual information which serves as a basis for correct recognition judgments. In the case of the present experiment, it could be argued that the attempt was made to introduce a semantic bias at input: there should be, and there is, an effect of this information on the recognition test. However, information about the relevant semantic sense constitutes only one aspect of the information necessary for rendition of accurate recognition judgments. Therefore, provision of semantic information should, and does, have only limited effectiveness as a retrieval cue. While this interpretation is admittedly post hoc, the approach seems much more compatible with our data than the widely held alternative view that, once the relevant semantic sense is activated, the necessary contextual or occurrence information is mechanically accessed.

\section{REFERENCES}

ANderson, J. R., \& Bower, G. H. Recognition and retrieval processes in tree recall. Psychological Review, 1972, 79. 97.123.

ANDERSON, J. R., \& Bower, G. H. A propositional theory of recognition memory. Memory \& Cognition, 1974, 2, 406-412.

Barnhart, C. L. (Ed.) American college dictionary. New York: Random. 1959.

Bevan, W., Dukes, W. F., \& Avant, L. The effect of variation in specific stimuli on memory for their superordinates. American Joumal of Psychology, 1966, 79, 250-257.

Hintzman, D. L. Theoretical implications of the spacing effect. In R. K. Solso (Ed.), Theories in cognitive psychology: The Loyola symposium. Pontac, Md: Erlbaum, 1974.

Hintzman, D. L., \& Block, R. A. Repetition and memory: Evidence for a multiple-trace hypothesis. Journal of Experimental Psychology, 1971, 88, 297-306.

Light, L. L., \& Carter-Sobell, L. Effects of changed semantic context on recognition memory. Journal of Verbal Learning and Verbal Behavior, 1970, 9, 1-11.

Light, L., Kimble, G. A., \& Pellegrino, J. W. Comments on episodic memory: When recognition fails. Journal of Experimental Psychology: General, 1975, 104, 30-36.

Madigan, S. A. Intraserial repetition and coding processes in free recall. Journal of Verbal Learning and Verbal Behavior, 1969, 8, 828-835.

Martin, E. Generation-recognition retrieval theory and the encoding specificity principle. Psychological Review, 1975, 82, 150-153

McCormack, P. D., \& Swenson, A. L. Recognition memory for rare and common words. Joumal of Experimental Psychology. 1972. 95, 72-77.

Melton, A. W. Repetition and retrieval from memory. Science, 1967, 158. 532.

Melton, A. W. The situation with respect to the spacing of repetitions and memory. Joumal of Verbal Learning and Verbal Behavior, 1970, 9. 596-606.

Murdock. B. B., JR. An analysis of the recognition process. In C. N. Cofer \& B. S. Musgrave (Eds.), Verbal behavior and learning: Problems and processes. New York: McGrawHill. 1963. Pp. 10-32.

Perfetti. C. A., \& Lindsey, R. Polysemy and memory. Journal of Psycholinguistic Research, 1974, 3, 75-89.

Reder. L. M., Anderson, J. R., \& Bjork, R. A. A semantic interpretation of encoding specificity. Journal of Experimental Psychology, 1974. 102, 648-656.

Tulving, E., \& Thomson, D. M. Encoding specificity and retrieval processes in episodic memory. Psychological Review, 1973, 80, 352.373.

Underwood, B. J., Zimmerman, J., \& Freund, J. S. Retention of frequency information with observations on recognition and recall. Joumal of Experimental Psychology, 1971, 87, 149-162.

Watkins, M. J.. \& Tulving. E. Episodic memory: When recognition fails. Joumal of Experimental Psychology: General, 1975, 104, 5-29.

Winograd, E.. \& Geis, M. F. Semantic encoding and recognition memory; A test of encoding variability theory. Journal of Experimental Psychology, 1974, 102, 1061-1068.

Winograd. E.. \& Raines, S. R. Semantic and temporal variation in recognition memory. Journal of Verbal Learning and Verbal Behavior, 1972. 11, 114-119.

(Received for publication November 26, 1975; revision accepted February 25.1976.$)$ 